\title{
Donor-Derived T-Cell Large Granular Lymphocytic Leukemia in a Patient With Peripheral T-Cell Lymphoma
}

\author{
Juliana E. Hidalgo Lopez, MDa,; ; Mariko Yabe, MD, PhD a,*; Adrian A. Carballo-Zarate, MDa; \\ Sa A. Wang, MD; ; Jeffrey L. Jorgensen, MD, PhD; ; Sairah Ahmed, MD ; John Lee, MD, PhD; \\ Shaoying Li, MDª Ellen Schlette, MD; ${ }^{a}$ Timothy McDonnell, MD, PhDa; Roberto N. Miranda, MD; \\ L. Jeffrey Medeiros, $\mathrm{MD}^{\mathrm{a}}$; Carlos E. Bueso-Ramos, $\mathrm{MD}, \mathrm{PhD}^{\mathrm{a}}$; and C. Cameron Yin, MD, $\mathrm{PhD}^{\mathrm{a}}$
}

\begin{abstract}
T-cell large granular lymphocytic (T-LGL) leukemia after hematopoietic stem cell transplantation (SCT) is rare and its natural history and clinical outcome have not been well described. We report the clinical, morphologic, immunophenotypic, and molecular features of a case of donor-derived T-LGL leukemia in a 16-year-old man who received allogeneic SCT for peripheral T-cell lymphoma not otherwise specified (PTCL-NOS). The patient presented with persistent neutropenia and splenomegaly 9 months after SCT when the chimerism study showed a $100 \%$ donor pattern. A splenectomy revealed T-LGL leukemia. Flow cytometric analysis showed an aberrant T-cell population positive for CD3, CD5 (dim, subset), CD7, CD8, CD16 (subset), CD57, CD94 (dim, partial), and T-cell receptor (TCR) $\alpha \beta$, and negative for CD4, CD26, CD56, and TCR $\gamma \delta$. Molecular studies showed monoclonal TCR $\beta$ and TCR $\gamma$ gene rearrangements. Both the immunophenotype and molecular profile of the T-LGL leukemia were different from the pre-SCT PTCL. Sequencing analysis for STAT3 exon 21 did not reveal any mutation in both pre-SCT and post-SCT specimens. The patient did not receive any treatment for T-LGL leukemia; however, his count progressively increased after splenectomy, despite the presence of persistent T-LGL leukemia in the bone marrow. There was no evidence of recurrent PTCL. We propose an algorithm to diagnose this rare post-SCT neoplasm.
\end{abstract}

J Natl Compr Canc Netw 2016;14(8):939-944

T-cell large granular lymphocytic (T-LGL) leukemia is an indolent neoplasm characterized by a persistent (>6 months) increase in peripheral blood LGLs, usually between 2 and $20 \times 10^{9} / \mathrm{L} .{ }^{1}$ Patients often present with neutropenia, splenomegaly, and autoimmune disorders. The LGLs are typically cytotoxic $\mathrm{T}$ cells expressing CD3, CD8, and T-cell receptor (TCR) $\alpha \beta$, with monoclonal TCR gene rearrangements. The pathogenesis of T-LGL leukemia is not well defined. However, chronic antigenic stimulation has been suggested as one underlying mechanism because of the frequent association between T-LGL leukemia and autoimmune disorders. ${ }^{2}$

\footnotetext{
From the Departments of a Hematopathology and bStem Cell

Transplantation, The University of Texas MD Anderson Cancer Center, Houston, Texas.

*These authors contributed equally to this work.

Submitted March 11, 2016; accepted for publication May 17, 2016.
}

T-LGL proliferation has been observed after allogeneic and autologous hematopoietic stem cell transplantation (SCT)..$^{3-6}$ The expanded T-LGLs are usually polyclonal or oligoclonal. Rare cases of T-LGL leukemia have also been reported after hematopoietic SCT, ${ }^{7-12}$ with a frequency of $0.5 \% .^{12}$ Whereas most patients with T-LGL leukemia occurring in the postSCT setting had remained asymptomatic with stable LGL counts and did not require specific treatment, 9,12 rare patients have been reported with an aggressive clinical course with progressive pancytopenia and death in a short interval. ${ }^{8}$ 
We report a case of a young patient with a history of peripheral T-cell lymphoma not otherwise specified (PTCL-NOS) who developed T-LGL leukemia of donor origin after allogeneic SCT, with a comprehensive literature review. A diagnostic algorithm to distinguish T-LGL leukemia and T-LGL proliferation and other lymphoproliferative disorders after SCT is proposed.

\section{Case Report}

A 16-year-old man initially presented to another hospital with fever, weight loss, lymphadenopathy, and hepatosplenomegaly in October 2012. A diagnosis of PTCL-NOS was established based on an inguinal lymph node (LN) biopsy. He came to our hospital for a second opinion and treatment options, where we confirmed the diagnosis of PTCL-NOS associated with hemophagocytosis and bone marrow (BM) involvement. He received dexamethasone, methotrexate, ifosfamide, L-asparaginase, and etoposide (SMILE) for 6 cycles beginning in January 2013. At the end of this regimen, PET scan showed increased FDG activity in the spleen. He was then treated with 2 cycles of cyclophosphamide, vincristine, doxorubicin, and prednisolone (CHOP) followed by 2 cycles of gemcitabine and 2 doses of romidepsin due to persistent disease in the BM. At this time, he received 2 cycles of alemtuzumab with a reduction of tumor burden in the BM (partial response). On June 4, 2014, he received allogeneic SCT from his brother with gemcitabine, clofarabine, and busulfan. Unfortunately, he developed primary graft failure. He then received a second transplant from his brother using fludarabine, antithymocyte globulin (ATG), and a higher CD34 cell dose in July 2014, which was successfully engrafted as shown by $100 \% \mathrm{~T}$ cells and myeloid cells of donor origin on microsatellite polymorphism analysis. He experienced complete remission of PTCL.

Three months later, his clinical course was complicated by Epstein-Barr virus (EBV)-positive posttransplant lymphoproliferative disorders (PTLD) in the duodenum and supraclavicular LN that were treated with rituximab and resulted in complete remission. However, he remained pancytopenic and did not respond to granulocyte colony-stimulating factor and was not explained by viral infection (negative EBV and cytomegalovirus [CMV] by PCR), medication, chimerism status (100\% engraftment), or BM involvement by neoplasm. A CBC count showed a WBC of $3.1 \mathrm{~K} / \mathrm{mcL}$ (reference range, 5.1$15.5 \mathrm{~K} / \mathrm{mcL}$ ), hemoglobin level of $10 \mathrm{~g} / \mathrm{dL}$ (reference range, $9.5-14.8 \mathrm{~g} / \mathrm{dL}$ ), and platelet count of $21 \mathrm{~K} / \mathrm{mcL}$ (reference range, 159-353 K/mcL), with a differential count of $12 \%$ neutrophils (absolute neutrophil count, $0.37 \mathrm{~K} / \mathrm{mcL}$; reference range, $1.70-7.30 \mathrm{~K} / \mathrm{mcL}$ ), 73\% lymphocytes (absolute lymphocyte count, 2.25 $\mathrm{K} / \mathrm{mcL}$; reference range, $1.00-4.80 \mathrm{~K} / \mathrm{mcL}$ ), $9 \%$ monocytes, $2 \%$ eosinophils, $2 \%$ metamyelocytes, and $2 \%$ blasts. The serum lactate dehydrogenase level was 496 IU/L (reference range, 313-618 IU/L). $\beta_{2}$-microglobulin was not assessed. In addition, PET scan showed splenomegaly without increased FDG uptake. Therefore, spleen sequestration was thought to be the cause of pancytopenia. In April 2015, a splenectomy was performed, which revealed T-LGL leukemia. At this time, the patient was negative for cytomegalovirus (CMV) or EBV infection by PCR. No evidence of graft-versus-host disease (GVHD) was present. He did not receive any treatment for T-LGL leukemia; however, his WBC progressively increased to above normal, despite the presence of persistent T-LGL leukemia in the BM. At the last follow-up, 7 months after splenectomy, a CBC showed a WBC of $16.2 \mathrm{~K} / \mathrm{mcL}$, hemoglobin level of $13.8 \mathrm{~g} / \mathrm{dL}$, and platelet count of $405 \mathrm{~K} / \mathrm{mcL}$, with persistent T-LGL leukemia in the BM and peripheral blood. There was no evidence of recurrent PTCL or PTLD. The clinical course is summarized in Figure 1.

\section{Morphologic and Immunophenotypic Findings}

Histologic sections of the initial inguinal LN biopsy showed altered LN architecture with regressed follicles. The paracortex was expanded by atypical cells with variable size, irregular nuclear contours, coarse chromatin, occasional conspicuous nucleoli, and moderate amount of cytoplasm. Immunostains revealed that the atypical cells were mainly $\mathrm{CD} 3+\mathrm{T}$ cells with decreased CD7 expression. A BM biopsy showed an atypical interstitial lymphoid infiltrate composed predominantly of small to intermediatesized cells positive for granzyme B and TIA-1, supporting a diagnosis of PTCL-NOS (Figure 2AC). In situ hybridization for EBV-encoded small RNA (EBER) was negative. Flow cytometric analysis 


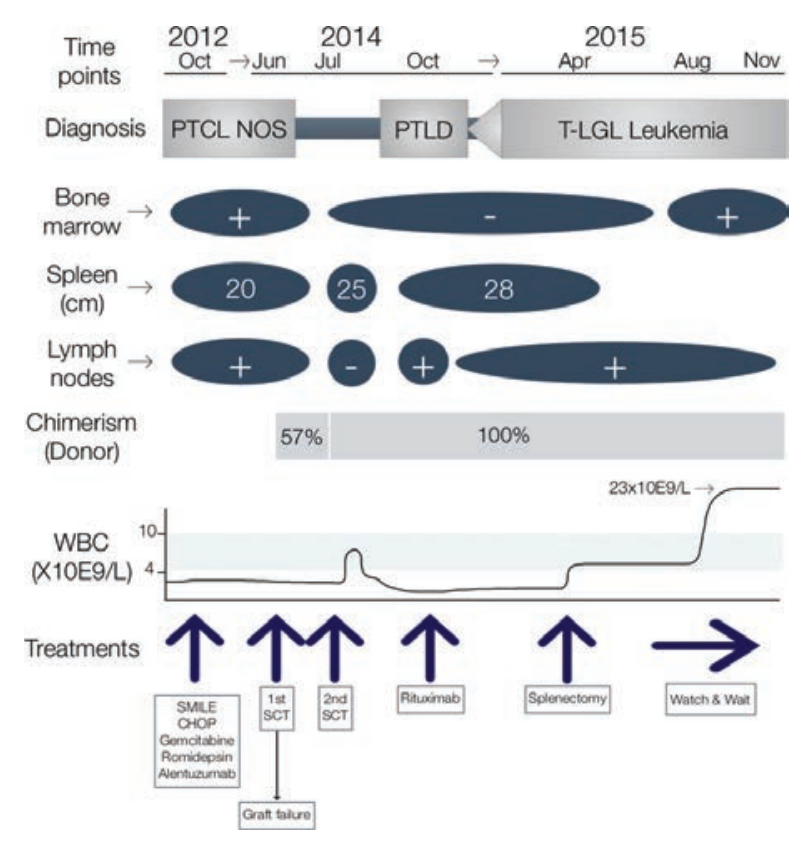

Figure 1. Clinical course. The time points when each diagnosis was made, and the hematopoietic organs involved, chimerism status, WBC count, and treatments received are listed.

Abbreviations: CHOP, cyclophosphamide, vincristine, doxorubicin and prednisolone; PTCL NOS, peripheral T-cell lymphoma not otherwise specified; PTLD, post-transplant lymphoproliferative disorder; SCT, stem cell transplantation; SMILE, dexamethasone, methotrexate, ifosfamide, L-asparaginase and etoposide; T-LGL, T-cell large granular lymphocytic.

of the pretransplant BM showed an aberrant T-cell population positive for CD2, CD3, CD5 (subset), CD8, CD26, CD57 (partial), and TCR $\alpha \beta$, and negative for CD4, CD7, CD16, CD56, CD94, and TCR $\gamma \delta$ (Figure 2D-F).
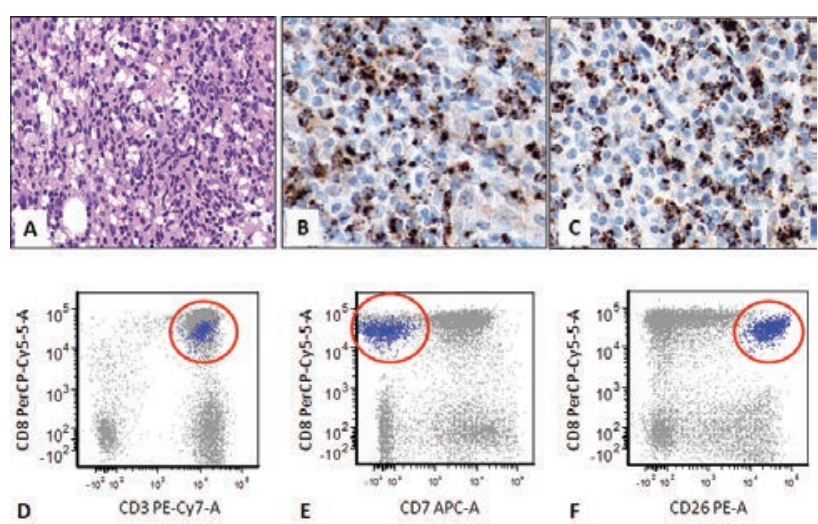

Figure 2. Morphologic and immunophenotypic features of peripheral T-cell lymphoma not otherwise specified. (A) The bone marrow biopsy is hypercellular with an atypical interstitial infiltrate of predominantly small to intermediate-sized cells (hematoxylin-eosin, original magnification $\times 200$ ). Immunostains show that these atypical cells are positive for (B) granzyme B (original magnification x200) and (C) TIA-1 (original magnification $\times 200$ ). Flow cytometry analysis shows an aberrant T-cell population that is (D) positive for CD3 and CD8, (E) negative for CD7, and (F) positive for CD26.
The duodenum biopsy showed a polymorphous lymphoid infiltrate in the mucosa composed of small to intermediate-sized lymphocytes, plasmacytoid lymphocytes, plasma cells and immunoblasts. Immunostains revealed a mixed population of $\mathrm{B}$ cells and $\mathrm{T}$ cells, with occasional scattered CD30+ cells. A stain for EBER was positive. These findings support a diagnosis of PTLD, polymorphic type. The supraclavicular LN showed a complete effacement of the nodal architecture. The paracortex was expanded by sheets of plasma cells admixed with small to intermediate-sized lymphocytes and occasional large atypical lymphocytes. The plasma cells were lambda light chain restricted. A stain for EBER was positive. These findings support a diagnosis of PTLD, monomorphic type.

The spleen showed expanded red pulp infiltrated by numerous small lymphoid cells with hyperchromatic nuclei and moderate to abundant cytoplasm positive for CD3, granzyme B, and TIA1 (Figure 3A-C). Flow cytometric analysis of the spleen and a subsequent BM showed an aberrant T-cell population positive for CD2, CD3, CD5 (dim, subset), CD7, CD8, CD16 (subset), CD57, CD94 (dim, partial), and TCR $\alpha \beta$, and negative for CD4, CD10, CD25, CD26, CD56, and TCR $\gamma \delta$, consistent with T-LGLs (Figure 3D-F). This immunophenotype differed from the pretransplant PTCL in the expression of CD7, CD16, CD26, CD57, and CD94. Interestingly, a retrospective review of the flow cytometry result from the supraclavicular LN revealed a small population of aberrant T-LGLs with the same immunophenotype, suggesting that, in addition to PTLD, this LN was also involved by T-LGL leukemia.

\section{Molecular and Cytogenetic Findings}

Molecular studies using PCR/capillary electrophoresis of a pretransplant BM demonstrated monoclonal TCR $\gamma$ and TCR $\beta$ rearrangements, with clonal peaks at sizes 146 and 179 for TCR $\gamma$, and 260 for TCR $\beta$. Molecular analysis of the spleen also showed monoclonal TCR $\gamma$ and TCR $\beta$ rearrangements; however, the predominant clonal peaks were 172 for TCR $\gamma$ and 250 for TCR $\beta$ (Figure 4). In accordance with flow cytometry findings, we retrospectively detected a low-level TCR $\gamma$ peak with size 172 and TCR $\beta$ peak with size 250 in the supraclavicular LN specimen. 

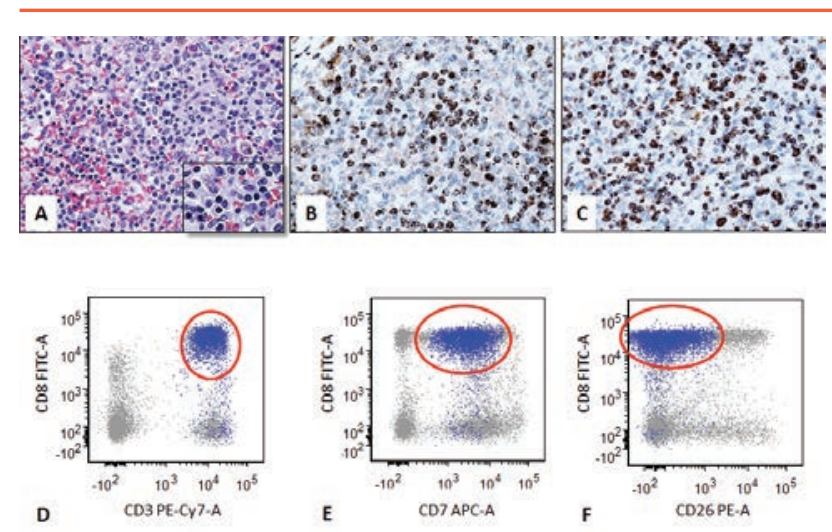

Figure 3. Morphologic and immunophenotypic features of T-cell large granular lymphocyte leukemia. (A) Histologic sections of the spleen show expanded red pulp that is infiltrated by numerous smal lymphoid cells (hematoxylin-eosin, original magnification $x 400$ ). At a higher magnification, the cells show round hyperchromatic nuclei and moderate to abundant cytoplasm (inset, original magnification $x 1,000)$. Immunostains reveal that the lymphoid cells are positive for (B) granzyme B (original magnification $\times 400$ ) and (C) TIA-1 (original magnification $\mathrm{x} 400$ ). Flow cytometry analysis shows an aberrant T-cell population that is (D) positive for CD3 and CD8, (E) positive for CD7, and (F) negative for CD26. This immunophenotype differs in the expression pattern of CD7 and CD26 compared with the pretransplant sample shown in Figure 2.

We performed mutation analysis for STAT3 exon 21 using PCR/Sanger sequencing on 4 specimens: a pre-SCT BM (involved by PTCL), a post-SCT LN, spleen, and BM (involved by T-LGL leukemia). However, no mutation was detected in any of the 4 specimens (see supplementary eAppendix 1, available with this article at JNCCN.org).

Conventional cytogenetic analyses of a pretransplant BM and the posttransplant spleen and BM showed a normal diploid karyotype, 46,XY[20].

\section{Discussion}

Expansion of T-LGLs after hematopoietic SCT has been well described. Most of these T-LGLs are reactive in nature with a self-limiting clinical course and patients are usually asymptomatic. ${ }^{3-6}$ However, rare cases of T-LGL leukemia after hematopoietic SCT have been reported in single case reports ${ }^{7-11}$ or small case series. ${ }^{12}$ Gill et a ${ }^{12}$ reported 7 cases of T-LGL leukemia among 1,675 consecutive cases of allogeneic and autologous hematopoietic SCT in a 20-year period, representing a frequency of $0.5 \%$. These authors suggested that T-LGL leukemia in posttransplant patients may have a different clinical course and pathogenesis compared with de novo T-LGL leukemia. Patients often remained stable for years and did not require specific treatment. ${ }^{12}$

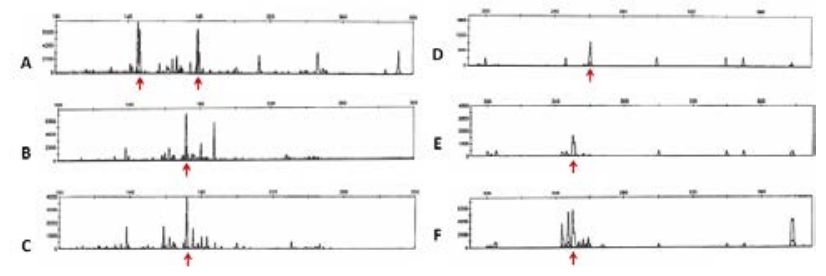

Figure 4. Molecular analysis of T-cell receptor (TCR) $\gamma(A-C)$ and TCR $\beta$ (D-F) gene rearrangements. Molecular studies using PCR followed by capillary electrophoresis of a pretransplant bone marrow demonstrate both clonal TCR $\gamma$ and TCR $\beta$ gene rearrangements, with clonal peaks at sizes 146 and 179 for TCR $\gamma(A)$, and 260 for TCR $\beta$ (D). Molecular analyses of the spleen and a subsequent bone marrow also show monoclonal TCR $\gamma$ and TCR $\beta$ gene rearrangements; however, the predominant clonal peaks are 172 for $\operatorname{TCR} \gamma(B, C)$ and 250 for $\operatorname{TCR} \beta(E, F)$.

The pathogenesis of T-LGL expansion and leukemia is not well understood. Most studies have suggested a causal relation to chronic antigenic stimulation due to viral infection, autoimmune diseases, GVHD, or immunosuppression with decreased immunosurveillance, leading to T-LGL proliferation or neoplastic transformation. ${ }^{5,6,13}$ Kim et $\mathrm{al}^{6}$ reported a strong association of LGL lymphocytosis with chronic GVHD, pretransplant CMV status of the recipient, and CMV antigenemia. Mohty et $\mathrm{al}^{4}$ reported that T-LGL proliferations occurred more frequently after a reduced fludarabine and ATG-based preparative regimen, because high immunosuppression of the host's immunity may lead to a state of immune balance favorable to the emergence of specific $T$ subsets, such as LGL. Similarly, our patient received fludarabine and an ATG-based preparative regimen for his SCT. Furthermore, it has been reported that T-LGL lymphocytosis in the post-SCT setting is associated with better overall survival and lower rate of disease relapse, suggesting that T-LGL might be a subset of effector lymphocytes that may contribute to the graft-versus-tumor effect. ${ }^{4,6}$ The patient was negative for CMV at the time of the development of T-LGL leukemia. He did not have GVHD. However, his CMV IgG antibodies were positive in the preSCT workup. This finding is in keeping with the hypothesis that the T-LGL proliferation could be related to $\mathrm{CMV}$ in the context of chronic antigen stimulation.

Recently, somatic mutations of STAT3 have been described in $40 \%$ to $70 \%$ cases of T-LGL leukemia. ${ }^{14,15}$ These mutations occur at the Src homology 2 (SH2) domain of STAT3, which mediates dimerization and activation of the STAT3 
protein, suggesting that aberrant STAT3 signaling underlies the pathogenesis of this disease. ${ }^{14}$ STAT3 mutations constitutively activate the transcription of a STAT3 isoform, which is responsible for IL-6dependent T-cell proliferation, and upregulate the downstream target genes of the STAT3 pathway, including IFNGR2, BCL2L1, and JAK2. STAT3 mutations are associated with increased phosphorylated STAT3 protein, which can be visualized by immunohistochemistry. More recently, Liang et $a{ }^{16}$ showed that phosphorylated STAT3 was expressed in hematopoietic SCT-associated T-LGL leukemia by immunohistochemistry. As a result, specific STAT3 inhibitors have been evaluated as a novel therapeutic regimen for the treatment of T-LGL leukemia. ${ }^{17}$

Determination of the actual frequency of T-LGL leukemia in the post-hematopoietic SCT setting has been challenged by the difficulty in the differential diagnoses between T-LGL proliferation and T-LGL leukemia. The published literature needs to be carefully reviewed because of the heterogeneity in terminology and diagnostic criteria. One study reported a frequency of $0.5 \% .^{12}$ Patients with T-LGL proliferation are usually asymptomatic and have disease that is transient, nonprogressive, selflimiting, and polyclonal or oligoclonal, whereas patients with T-LGL leukemia usually have a persistently increased number of T-LGLs with immunophenotypic aberrancy and monoclonal TCR rearrangements, and have the typical manifestations of T-LGL leukemia with cytopenia, recurrent infections, autoimmune disorders, and splenomegaly. Other causes of cytopenia in the post-SCT setting, such as transplant-related causes (graft failure, poor engraftment, GVHD), infections (CMV, EBV), medications (ganciclovir), and relapse of initial disease, also need to be considered. Recently, a high level of STAT3 mutation has been shown to have diagnostic value in distinguishing T-LGL leukemia from other mature $\mathrm{T}$-cell neoplasms and reactive conditions. We did not detect any mutation in exon 21 of the STAT3 gene in this case. Nevertheless, the morphologic, immunophenotypic, and molecular findings support a diagnosis of T-LGL leukemia.

The current case report complements the few reports of T-LGL leukemia in the post-SCT setting reported previously. The morphologic features of T-LGL leukemia can be subtle, and

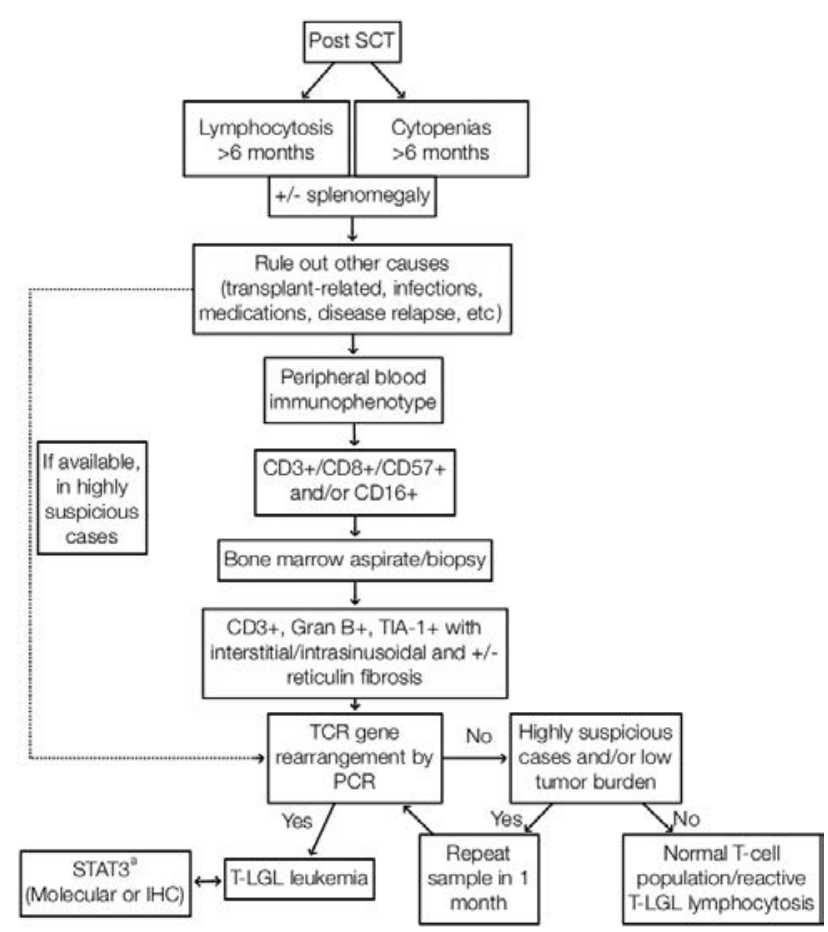

Figure 5. Diagnostic algorithm for the differential diagnosis of T-LGL leukemia and T-LGL proliferation after stem cell transplantation. Abbreviations: IHC, immunohistochemistry; SCT, stem cell transplantation; T-LGL, T-cell large granular lymphocytic; TCR, T-cell receptor. aPresent in $30 \%-70 \%$ of T-LGL leukemia.

the cytopenia accompanying T-LGL leukemia also can be attributable to other complications of SCT. Therefore, this disease might be missed or interpreted as recurrence of the initial disease. It is important for hematopathologists and hematologists to be aware of this entity in order to guide treatment strategies. We propose a diagnostic algorithm for the differential diagnoses of T-LGL leukemia and T-LGL proliferation after SCT (Figure 5). The most important distinguishing features are TCR gene rearrangements and STAT3 mutation status.

\section{References}

1. Chan WC, Foucar K, Morice WG, et al. T-cell large granular lymphocytic leukemia. In: Swerdlow SH, Campo E, Harris NL, et al, eds. WHO Classification of Tumours of Haematopoietic and Lymphoid Tissues. Lyon, France: IARC; 2008:272-273.

2. Zambello R, Semenzato G. Large granular lymphocyte disorders: new etiopathogenetic clues as a rationale for innovative therapeutic approaches. Haematologica 2009;94:1341-1345.

3. Dolstra H, Preijers F, Van de Wiel-van Kemenade E, et al. Expansion of CD8+CD57+ T cells after allogeneic BMT is related with a low incidence of relapse and with cytomegalovirus infection. Br J Haematol 1995;90:300307.

4. Mohty M, Faucher C, Vey N, et al. Features of large granular lymphocytes (LGL) expansion following allogeneic stem cell transplantation: a longterm analysis. Leukemia 2002;16:2129-2133. 
Hidalgo Lopez et al

5. Nann-Rutti S, Tzankov A, Cantoni N, et al. Large granular lymphocyte expansion after allogeneic hematopoietic stem cell transplant is associated with a cytomegalovirus reactivation and shows an indolent outcome. Biol Blood Marrow Transplant 2012;18:1765-1770.

6. Kim D, Al-Dawsari G, Chang $\mathrm{H}$, et al. Large granular lymphocytosis and its impact on long-term clinical outcomes following allo-SCT. Bone Marrow Transplant 2013;48:1104-1111.

7. Wong KF, Yip SF, So CC, et al. Cytomegalovirus infection associated with clonal proliferation of T-cell large granular lymphocytes: causal or casual? Cancer Genet Cytogenet 2003;142:77-79.

8. Au WY, Lam CC, Lie AK, et al. T-cell large granular lymphocyte leukemia of donor origin after allogeneic bone marrow transplantation. Am J Clin Pathol 2003;120:626-630.

9. Narumi H, Kojima K, Matsuo Y, et al. T-cell large granular lymphocytic leukemia occurring after autologous peripheral blood stem cell transplantation. Bone Marrow Transplant 2004;33:99-101.

10. Chang $H$, Kamel-Reid $S$, Hussain $\mathrm{N}$, et al. T-cell large granular lymphocytic leukemia of donor origin occurring after allogeneic bone marrow transplantation for B-cell lymphoproliferative disorders. Am J Clin Pathol 2005;123:196-199.
11. Kusumoto S, Mori S, Nosaka K, et al. T-cell large granular lymphocyte leukemia of donor origin after cord blood transplantation. Clin Lymphoma Myeloma 2007;7:475-479.

12. Gill H, Ip AH, Leung $R$, et al. Indolent T-cell large granular lymphocyte leukaemia after haematopoietic SCT: a clinicopathologic and molecular analysis. Bone Marrow Transplant 2012;47:952-956.

13. Bareau B, Rey J, Hamidou M, et al. Analysis of a French cohort of patients with large granular lymphocyte leukemia: a report on 229 cases. Haematologica 2010;95:1534-1541.

14. Koskela HL, Eldfors S, Ellonen P, et al. Somatic STAT3 mutations in large granular lymphocytic leukemia. N Engl J Med 2012;366:1905-1913.

15. Fasan A, Kern W, Grossmann V, et al. STAT3 mutations are highly specific for large granular lymphocytic leukemia. Leukemia 2013;27:1598-1600.

16. Liang CS, Quesada AE, Goswami M, et al. Phosphorylated STAT3 expression in hematopoietic stem cell transplant-associated large granular lymphocytic leukemia. Bone Marrow Transplant 2016;51:741-743.

17. Bilori $B$, Thota $S$, Clemente $M J$, et al. Tofacitinib as a novel salvage therapy for refractory T-cell large granular lymphocytic leukemia. Leukemia 2015;29:2427-2429.

\section{See JNCCN.org for supplemental online content.}

